\title{
Deterministic and Probabilistic Investigation on Multiple Crack Interactions in a Semi-Infinite Domain
}

\author{
Eui-Keun Kim, ${ }^{1}$ Habeun Choi, ${ }^{1}$ Kyoungsoo Park, ${ }^{1}$ and Won-Hee Kang ${ }^{2}$ \\ ${ }^{1}$ Department of Civil and Environmental Engineering, Yonsei University, 50 Yonsei-ro, Seodaemun-gu, \\ Seoul 120-749, Republic of Korea \\ ${ }^{2}$ Institute for Infrastructure Engineering, University of Western Sydney, Penrith, NSW 2751, Australia \\ Correspondence should be addressed to Won-Hee Kang; w.kang@uws.edu.au
}

Received 25 June 2015; Revised 15 July 2015; Accepted 22 July 2015

Academic Editor: Mohammed Nouari

Copyright (C) 2015 Eui-Keun Kim et al. This is an open access article distributed under the Creative Commons Attribution License, which permits unrestricted use, distribution, and reproduction in any medium, provided the original work is properly cited.

\begin{abstract}
The investigation of multiple crack interactions in fracture mechanics is important to predict the safety and reliability of structures. This study aims to investigate the interactions of multiple parallel cracks in a semi-infinite domain in both deterministic and probabilistic ways by using an automated finite element modeling procedure and the Monte Carlo simulation. The stress intensity factor is considered as an indicator of failure and accurately evaluated by using the domain integral technique. The variation of the stress intensity factor according to the position, the length, and the number of cracks is demonstrated. In a probabilistic investigation, the effects of the number of cracks, the random distribution of the crack lengths, and the crack interactions to the failure probability are studied for a semi-infinite domain. The stress redistribution among multiple cracks, the effect of unevenly distributed crack lengths, and the combined effect of crack length uncertainties and a crack shielding effect have been examined.
\end{abstract}

\section{Introduction}

The crack initiation, growth, and interaction of multiple cracks play a critical role in predicting structural failure in the fields of civil, electrical, and aerospace engineering. Multiple crack initiations and growth are generally observed in a structural member in service loading conditions because of initial defects, microstructures, and so forth. Such multiple cracks in a structural member significantly impact on the probability of failure due to uncertainties on crack interactions. Thus, a thorough investigation of multiple cracks in terms of their fracture mechanics is essentially needed to estimate the safety and reliability of structures, but such efforts have mainly been focused on in the investigations of a single crack or deterministic investigation of multiple cracks except several recent studies. For more realistic and reasonable research, the randomness or uncertainties in parameters should be considered but it is often hindered by the computational complexity in a probabilistic modeling of multiple cracks and uncertainties in fracture properties.

Multiple crack interactions have generally been investigated on the basis of deterministic approaches. The stress intensity factors of multiple cracks were evaluated for various configurations (e.g., [1-4]). For example, Tsang et al. [5] investigated multiple penny-shaped cracks in a solid elastic cylinder under mode-I loading and demonstrated the decrease of the stress intensity factor according to the increase of the number of parallel cracks. Loehnert and Belytschko [6] demonstrated crack shielding and amplification due to the interaction between multiple microcracks and a macrocrack. Kamaya [7] evaluated the stress intensity factor for various relative positions and shape of two semi-elliptical cracks. Liu et al. [8] analyzed multiple rock cracks under seepage pressure and addressed the interactions of multiple rock cracks.

Some recent studies on the probabilistic investigation and modeling of multiple cracks are found as follows. Xiaofeng et al. [9] developed a stochastic finite element model for plane multiple cracks based on the Taylor stochastic finite element method using the quarter point and triangular elements. The uncertainties in material properties, crack length, and load were considered. The partial derivatives of displacement and stiffness matrix with respect to random variables were derived using the Taylor stochastic finite element model, 


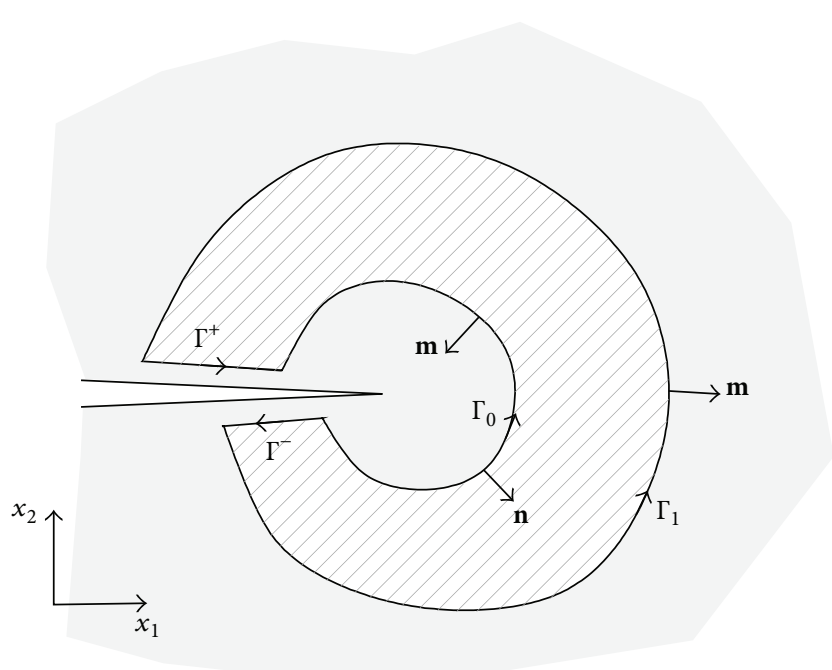

FIgURE 1: Contours around a crack tip for the domain integral.

and the partial descriptors of the stress intensity factor were obtained. Leonel et al. [10] conducted a probabilistic analysis on a multi-perforated panel with two cracks at each hole. The limit state was defined in terms of the number of load cycles. The uncertainties in loading, material properties, and geometric parameters were considered. The first-order reliability method and the response surface method were used in conjunction with the boundary element method. Feng et al. [11] proposed a probabilistic model for multiple crack growth in a stiffened panel in terms of crack sizes. The Monte Carlo simulation was utilized to estimate the partial descriptors of the crack growth model, and the correlation between the crack sizes was considered. The prediction of the crack propagation was modeled on the basis of the Paris-Erdogan law. Chowdhury et al. [12] evaluated the reliability of cracked specimens using the first- and secondorder reliability methods considering the uncertainty in crack geometry and material properties. Four linear elastic homogeneous isotropic cracked specimens with edge crack, double edge crack, edge crack with a fixed base, and an angled edge crack were considered. In addition, a shape sensitivity analysis of the stress intensity factor was conducted using the scaled boundary finite element method, which required no remeshing according to the size and orientation of a crack. However, these studies mainly focused on the methodological and theoretical development in probabilistic fracture mechanics modeling.

This study focuses on investigating the interaction effects of multiple parallel cracks on a semi-infinite domain. The investigation is conducted deterministically and probabilistically through integrating an automated finite element modeling procedure and the Monte Carlo simulation. The effects of the number of cracks and the randomness in the crack length to the stress intensity factor and the failure probability of the semi-infinite domain are investigated considering the crack shielding effect among multiple cracks.

\section{Evaluation of the Stress Intensity Factor}

For the evaluation of the stress state around a crack tip region, a stress intensity factor is introduced based on the linear elastic fracture mechanics. When a stress intensity factor is greater than a critical value, a crack propagates and the failure of a structure is expected for brittle materials. The critical value is generally called fracture toughness. The stress intensity factor is evaluated by employing various computational methods such as boundary element methods [13], quarter point elements [14, 15], domain integral [16], and generalized/extended finite element methods [17-19].

In the present study, the stress intensity factor at a crack tip is evaluated by utilizing the $J$-integral [20], which corresponds to the energy release rate, in conjunction with the domain integral technique [16]. The $J$-integral around a crack tip (see Figure 1) is defined along a contour $\left(\Gamma_{0}\right)$ with the normal vector $(\mathbf{n})$,

$$
J=\int_{\Gamma_{0}}\left(W \delta_{1 i}-\sigma_{i j} \frac{\partial u_{j}}{\partial x_{1}}\right) n_{i} d \Gamma,
$$

where $W$ is the strain energy density and $\sigma_{i j}$ and $u_{i}$ are the components of a stress tensor and a displacement vector in the Cartesian coordinates $\left(x_{1}-x_{2}\right)$ system. Alternatively, the $J$-integral is expressed along a closed contour $\left(\Gamma^{*}\right)$, which is expressed as

$$
\begin{aligned}
J= & \int_{\Gamma^{*}}\left(\sigma_{i j} \frac{\partial u_{j}}{\partial x_{1}}-W \delta_{1 i}\right) m_{i} q d \Gamma \\
& -\int_{\Gamma^{+}+\Gamma^{-}} \sigma_{2 j} \frac{\partial u_{j}}{\partial x_{1}} m_{2} q d \Gamma,
\end{aligned}
$$

where $q$ is an arbitrary smooth function that is unity on $\Gamma_{0}$ and zero on $\Gamma_{1}$ and $m_{i}$ is the components of the outward normal vector $(\mathbf{m})$ of the closed domain $\left(A^{*}\right)$, as shown in Figure 1. The closed contour $\left(\Gamma^{*}\right)$ consists of four curves of $\Gamma_{0}, \Gamma_{1}, \Gamma^{+}$, and $\Gamma^{-}$; that is, $\Gamma^{*}=\Gamma^{-}+\Gamma_{1}+\Gamma^{+}-\Gamma_{0}$. Through utilizing the divergence theorem, the contour integral expression (2) is converted to a domain integral [15]; that is,

$$
\begin{aligned}
J= & \int_{A^{*}} \frac{\partial\left(\sigma_{i j} \partial u_{j} / \partial x_{1}-W \delta_{1 i}\right) q}{\partial x_{i}} d A \\
& -\int_{\Gamma^{+}+\Gamma^{-}} \sigma_{2 j} \frac{\partial u_{j}}{\partial x_{1}} m_{2} q d \Gamma .
\end{aligned}
$$

Based on the equilibrium condition and eliminating thermal strain, body force, and crack face traction, one simplifies the domain integral expression as

$$
J=\int_{A^{*}}\left(\sigma_{i j} \frac{\partial u_{j}}{\partial x_{1}}-W \delta_{1 i}\right) \frac{\partial q}{\partial x_{i}} d A
$$

which is equivalent to (1).

The displacement and stress fields within a domain are evaluated by using a finite element method. A domain is discretized into a finite element mesh with quadratic 


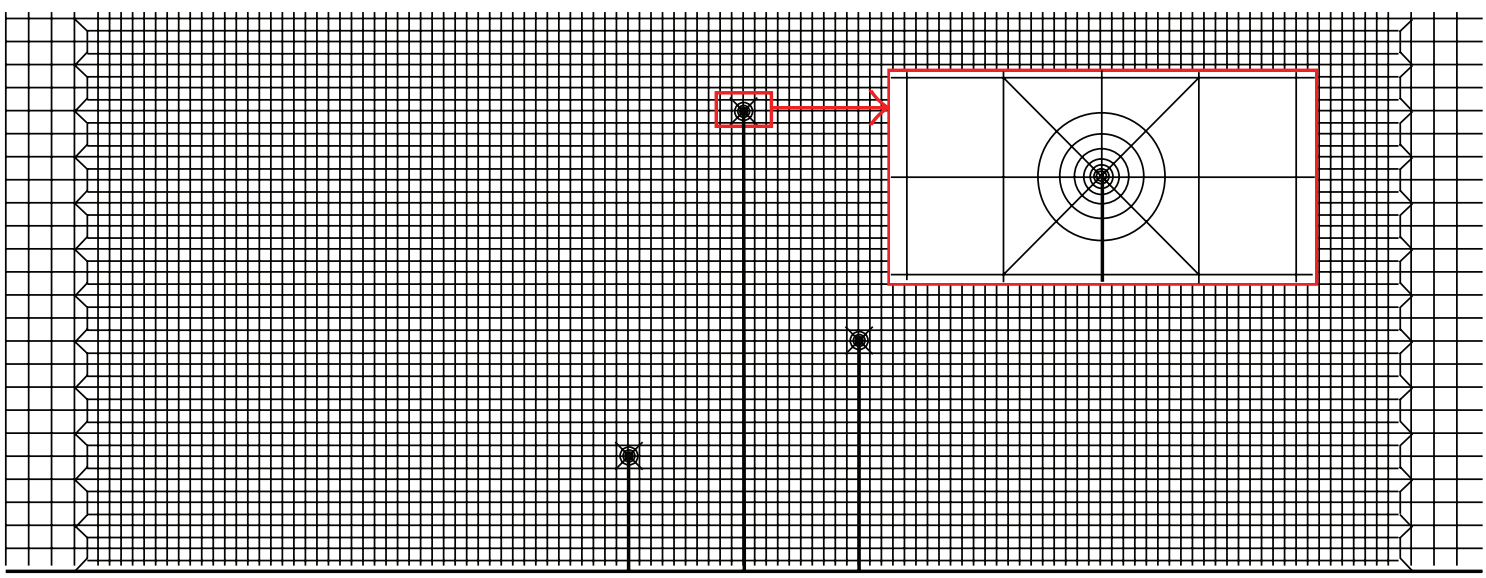

FIgURE 2: Finite element mesh around a crack tip region.

rectangle elements. A collapsed quarter point element is utilized around a crack tip region, which leads to a singular stress variation around a crack tip region. Figure 2 illustrates an example of a finite element mesh around a crack tip. The same discretization is employed for every crack tip region. Eight quarter point elements are used at a crack tip, and the size of element monotonically increases with the approximate ratio of 1.5 along the radial direction. For a significant amount of the finite element analysis with arbitrary multiple crack geometries in a semi-infinite domain, a finite element mesh is automatically generated by developing an in-house code with $\mathrm{C}$ language. In an in-house code, the position, the length, and the number of cracks are provided as input parameters. Then, elements along the specified cracks are removed, and nodes are duplicated along the cracks. Then, eight quarter point elements around crack tips are inserted at a crack tip, and then eight-node elements are inserted through monotonically varying element size, as shown in Figure 2 .

\section{Two-Crack Interactions}

When two cracks are placed in a domain, stress redistribution occurs, which results in the change of the stress intensity factor according to the position of cracks. In order to demonstrate such variations, two-crack interactions are first investigated for an infinite domain and a semi-infinite domain in this section. Then, three-crack interactions and more than three-crack interactions are addressed in Section 4.

3.1. Infinite Domain. For an infinite domain, the following two cases are considered: serial cracks and parallel cracks. First, two serial cracks are placed with the spacing $s$. The crack lengths of the two cracks are equally $2 a$, and remote tension $\left(\sigma_{\infty}\right)$ is applied. Then, the stress intensity factor is evaluated through changing the ratio of the spacing to the crack length $(s / 2 a)$ and nondimensionalized by dividing it by $\sigma_{\infty} \sqrt{\pi a}$. Note that $\sigma_{\infty} \sqrt{\pi a}$ is the stress intensity factor of a single crack with the length of $2 a$ in an infinite domain. Additionally, the nondimensionalized results are valid for any selection of the spacing ratio to the crack length because it is based on the linear elastic fracture mechanics. Figure 3(a) illustrates that the closer distance results in the stronger interaction, and thus the stress intensity factor increases further for both outer- and inner-crack tips $\left(C_{1}\right.$ and $\left.C_{2}\right)$. When the distance is large, the nondimensionalized stress intensity factor reaches unity, which corresponds to no interaction between the two cracks, that is, single crack case. The stress intensity factor at the outer-crack tip $\left(C_{1}\right)$ is lower than the factor at the innercrack tip $\left(C_{2}\right)$ because an additional serial crack adjacent to the inner-crack tip magnifies the stress concentration. On the other hand, when two cracks are placed in parallel with the spacing $s$, the stress intensity factor decreases while the spacing ratio decreases, as shown in Figure 3(b). Such reduction of the stress intensity factor is named the crack shielding effect. Additionally, the stress intensity factors of all the crack tips are identical because of the symmetry. In summary, the stress intensity factor is magnified for serial cracks while the stress intensity factor is reduced for a parallel crack. Then, one can expect that the formation of additional cracks is not always detrimental to a structure, and thus the failure probability can decrease due to the formation of multiple/additional cracks.

3.2. Semi-Infinite Domain. Two-crack interactions in a semiinfinite domain are investigated with remote tension $\left(\sigma_{\infty}\right)$. The geometry of a semi-infinite domain with two cracks is shown in Figure 4. For two cracks of $C_{1}$ and $C_{2}$, the lengths of two cracks are $a_{1}$ and $a_{2}$ with the spacing of the two cracks $\left(s_{12}\right)$, and the corresponding stress intensity factors are evaluated. Arbitrary combinations of two cracks in a semiinfinite domain are considered through employing the two variables: the ratio of a short crack length to a long crack length $\left(\gamma_{c}=\min \left(a_{1}, a_{2}\right) / \max \left(a_{1}, a_{2}\right)\right)$ and the ratio of the crack spacing to a long crack length $\left(\gamma_{s}=s_{12} / \max \left(a_{1}, a_{2}\right)\right)$. For five cases of $\gamma_{c}$, the stress intensity factor is evaluated through varying the spacing ratio $\left(\gamma_{s}\right)$ from 1.0 to 18 . The results in Figure 5 illustrate that the evaluated stress intensity factors of the two cracks are lower than the stress intensity factor of a single edge crack in a semi-infinite domain, that 


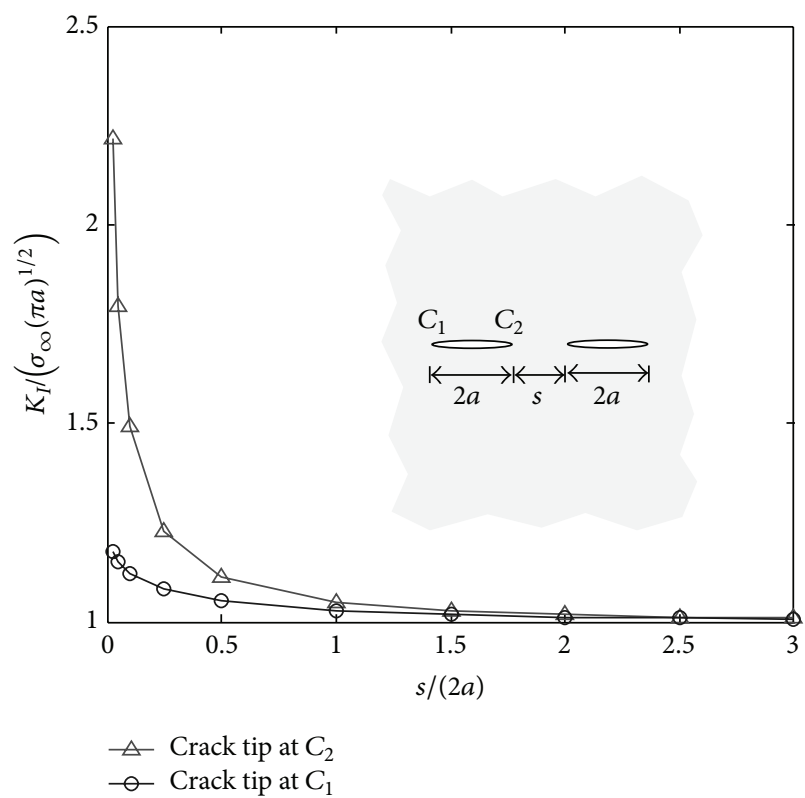

(a)

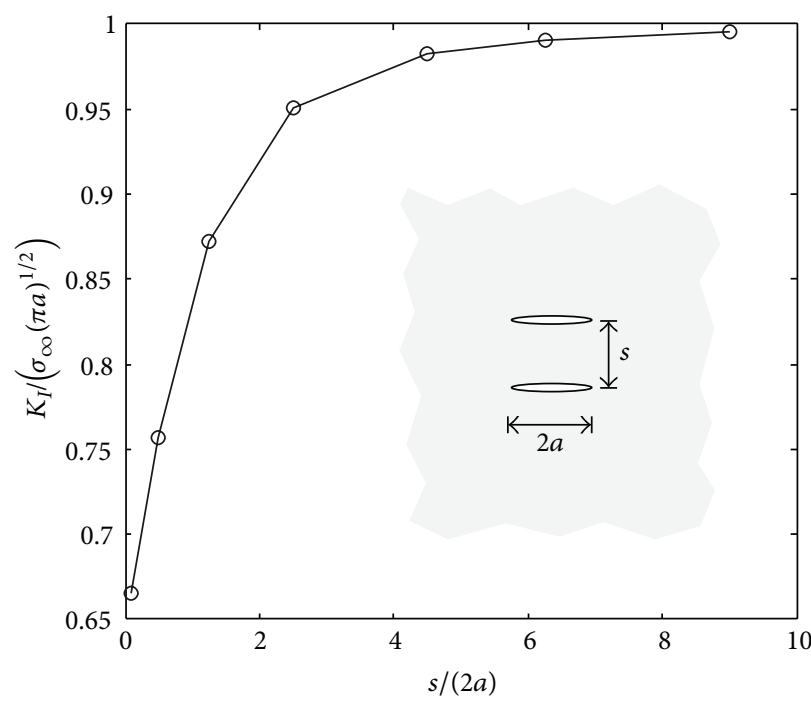

(b)

FIGURE 3: Nondimensionalized stress intensity factor of two cracks in an infinite domain for (a) serial cracks and (b) parallel cracks.

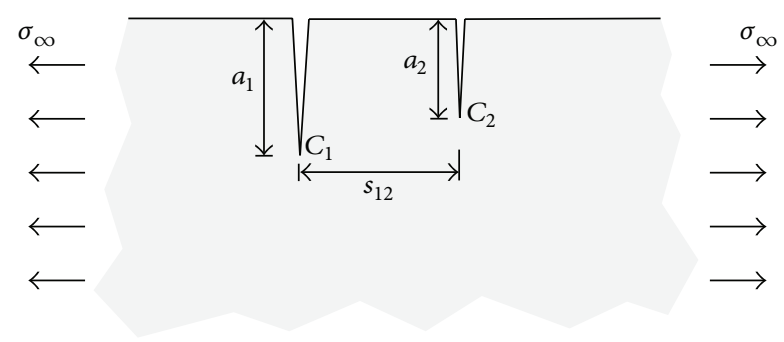

FIGURE 4: Geometry of two cracks in a semi-infinite domain.

is, $1.12 \sigma_{\infty} \sqrt{\pi a}$, for all cases because of the crack shielding effect. The decrease of the stress intensity factor of the longer crack tends to be more significant when the ratio of twocrack lengths $\left(\gamma_{c}\right)$ increases (Figure 5(a)). When two-crack lengths are similar (e.g., $\gamma_{c}=1.0$ ), the magnitude of stress concentration is similar. Thus, significant stress redistribution is expected, which lowers the stress intensity factor of a long crack compared to a single edge crack case. For example, when the lengths of the two cracks are the same $\left(a_{1}=a_{2}\right)$ and the spacing ratio is $1.0\left(\gamma_{s}=1\right)$, the normalized stress intensity factor is 0.857 , which is approximately $75 \%$ of the stress intensity factor for a single edge crack in a semi-infinite domain. If two-crack lengths are significantly different (e.g., $\gamma_{c}=0.25$ ), a long crack dominates the stress concentration, which is similar to the case of a single edge crack. In addition, when the spacing between two cracks increases, the stress intensity factor increases and converges to the value of $1.12 \sigma_{\infty} \sqrt{\pi a}$, which corresponds to the stress intensity factor of the single edge crack in a semi-infinite domain. The stress intensity factor of the shorter crack $\left(K_{I \min }\right)$ is lower than the stress intensity factor of a single crack case due to the existence of a longer crack (Figure 5(b)). The amount of reduction is more significant when the spacing ratio between two cracks $\left(\gamma_{s}\right)$ is closer, and the ratio of two-crack lengths $\left(\gamma_{c}\right)$ is smaller.

\section{Multiple Crack Interactions}

The effects of multiple cracks on the stress intensity factor in a semi-infinite domain are investigated through changing the spacing of cracks, the length of cracks, and the number of cracks. For $n$ cracks in a semi-infinite domain under remote tension, the stress intensity factor $\left(K_{I i}\right)$ of the $i$ th $\operatorname{crack}\left(C_{i}\right)$ is expressed as

$$
K_{I i}=\sigma_{\infty} \sqrt{\pi a_{i}} F_{i},
$$

where $a_{i}$ and $F_{i}$ are the length and the geometric function of the $i$ th crack, respectively. The spacing between the $i$ th crack and the $j$ th crack is denoted as $s_{i j}$. The geometric functions $\left(F_{i}\right)$ represent the change of the stress intensity factor according to the position, the length, and the number of cracks in a semi-infinite domain. In the following subsections, threecrack examples are first considered, and then the effects of the number of cracks are demonstrated through evaluating the normalized stress intensity factor, that is, $K_{I i} /\left(\sigma_{\infty} \sqrt{\pi a_{i}}\right)$.

4.1. Three-Crack Interactions. For three-crack interactions, the stress intensity factor of each crack is evaluated through varying the crack length ratio and the spacing ratio. First, the ratio of the 2 nd crack length to the 1st crack length $\left(a_{2} / a_{1}\right)$ ranges from 0 to 5 . Note that $a_{2} / a_{1}=0$ corresponds to the two-crack interactions of $a_{1}$ and $a_{3}$. The three cracks are equally spaced, and the ratio of the spacing between the two adjacent cracks to the 1st crack length is fixed to one (i.e., 


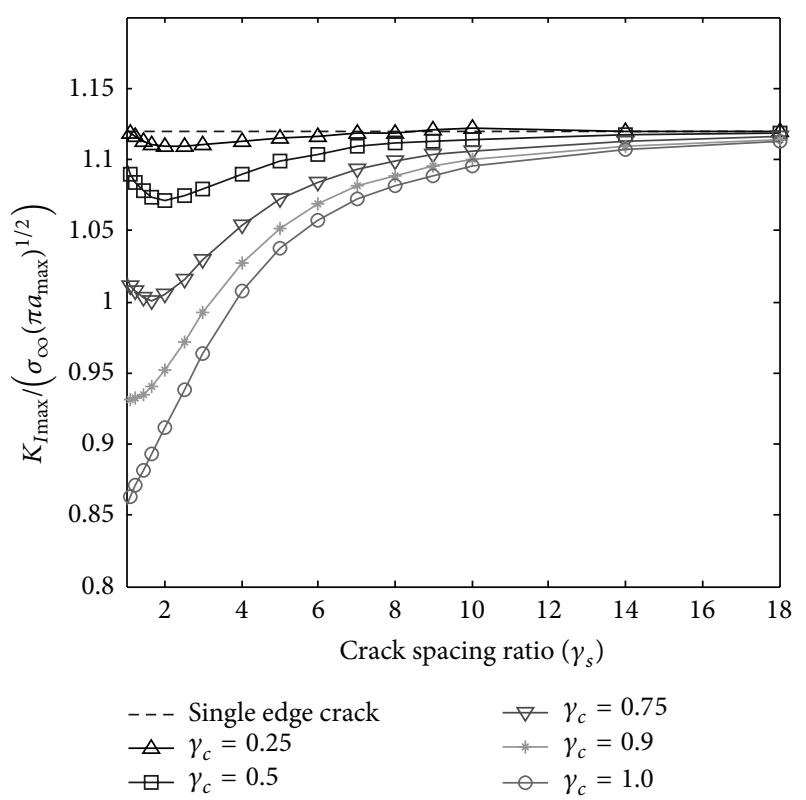

(a)

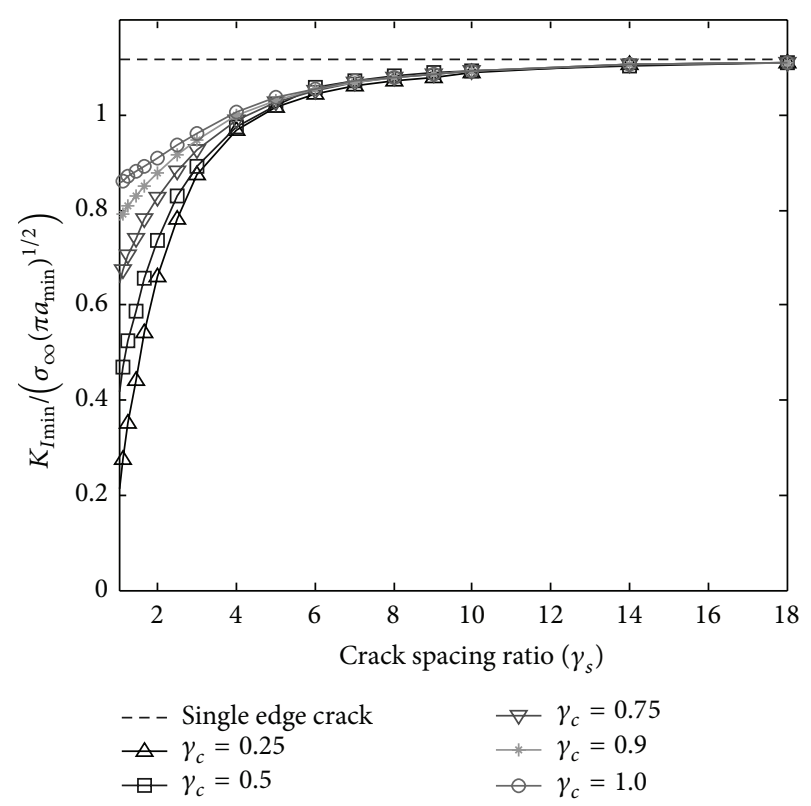

(b)

FIGURE 5: Nondimensionalized stress intensity factor of two cracks in a semi-infinite domain: (a) a long crack and (b) a short crack.

$\left.s_{12} / a_{1}=s_{23} / a_{1}=1\right)$. The results are illustrated in Figure 6 for three cases of the 3rd crack length ratio $\left(a_{3} / a_{1}=1\right.$, $2,3)$. As a guidance, the stress intensity factor of a single edge crack in a semi-infinite domain, that is, $1.12 \sigma_{\infty} \sqrt{\pi a}$, is plotted as a dashed line. The stress intensity factor of the 1st crack does not change significantly until the length of the second crack approximately reaches the length of the first crack (see Figure 6(a)). When the length of the second crack is greater than that of the first crack, the stress intensity factor tends to decrease because of the crack shielding effects. In addition, the greater length of the 3rd crack $\left(a_{3}\right)$ results in a more decrement in the stress intensity factor of the 1st crack, as expected. The stress intensity factor of the 2 nd crack increases and converges to that of the single edge crack in a semi-infinite domain, as the crack length of the 2nd crack increases (Figure 6(b)). When the 2nd crack length is relatively small, the compression on a crack surface is observed. This is because the crack opening width of the side cracks is larger than that of the center crack and thus the crack opening of the center crack is dominated by the interactions from the side cracks. For the 3rd crack, the stress intensity factor remains almost constant until the center crack reaches the length of the 3rd crack, which is similar to the 1 st crack case. In addition, when the three-crack lengths are identical, the normalized stress intensity factor of the side crack is higher than that of the center crack. This is because the center crack has more number of adjacent cracks than the side crack, and thus the stronger shielding effect is expected.

4.2. More than Three Cracks. Multiple crack interactions are investigated by changing the crack length ratio and the number of cracks. The second crack length ratio varies while the other crack length ratios to the length of the first crack are fixed as one. In addition, the crack spacing ratio to the first crack length is taken to be one. The number of cracks is considered as 3, 4, 5, 6, and 10. Figure 7 illustrates that the stress intensity factor of the first crack slightly decreases according to the increase of the number of cracks. When the 2nd crack length ratio $\left(a_{2} / a_{1}\right)$ is greater than 1.0, the difference of the stress intensity factors is smaller according to the change of the number of cracks. Additionally, the stress intensity factor of the 1st crack tip keeps decreasing as the stress intensity factor of the 2 nd crack tip converges to the value in the single crack example.

\section{Probabilistic Investigation of Multiple Crack Interactions}

5.1. Failure Probability of a Semi-Infinite Domain with Multiple Cracks. This section extends the discussions in the previous sections into the probabilistic investigation of multiple parallel cracks when the crack lengths are randomly distributed. For this purpose, the probability of failure of a semi-infinite domain is evaluated. When multiple cracks exist in a semiinfinite domain, the failure in a domain can be defined such that at least one crack has a stress intensity factor that exceeds the threshold value at its tip. This definition can be expressed using the following limit state function:

$$
\begin{aligned}
& g(\mathbf{X})=K_{I C}-\max \left(K_{I 1}(\mathbf{X}), K_{I 2}(\mathbf{X}), \ldots, K_{I i}(\mathbf{X}), \ldots,\right. \\
& \left.K_{I n}(\mathbf{X})\right),
\end{aligned}
$$

where $\mathbf{X}$ denotes a vector of parameters that include the length of all cracks and crack spacings in a semi-infinite domain. Additionally, $K_{I C}$ denotes the fracture toughness of a material, and $K_{I i}(\mathbf{X})$ denotes the stress intensity factor at 


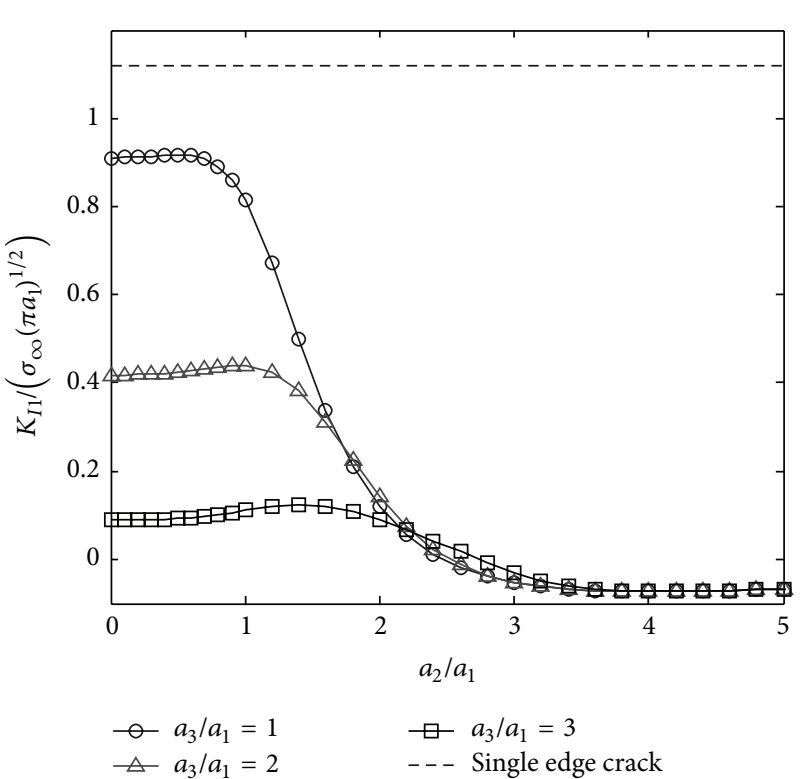

(a)

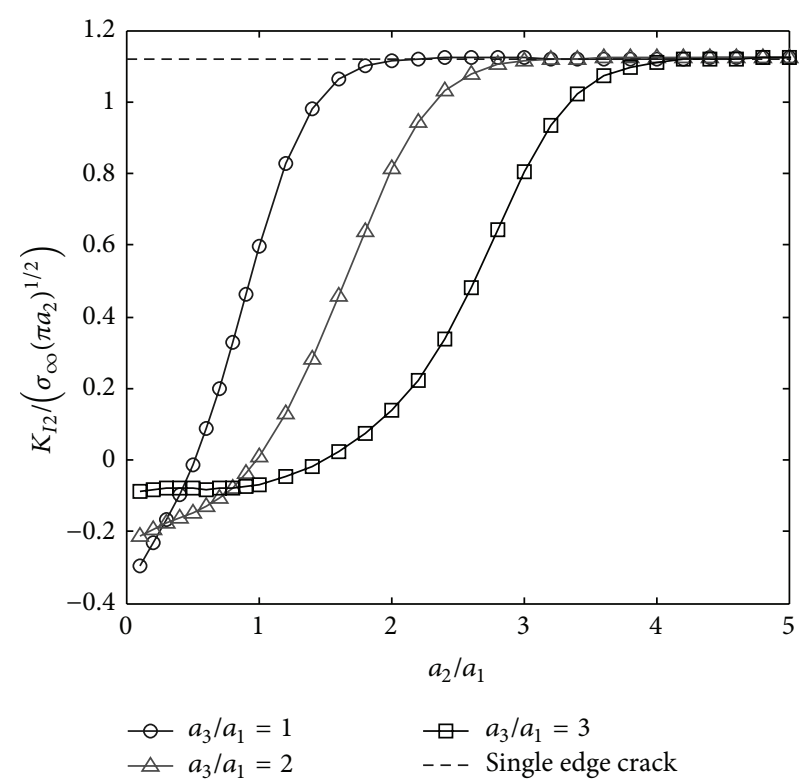

(b)

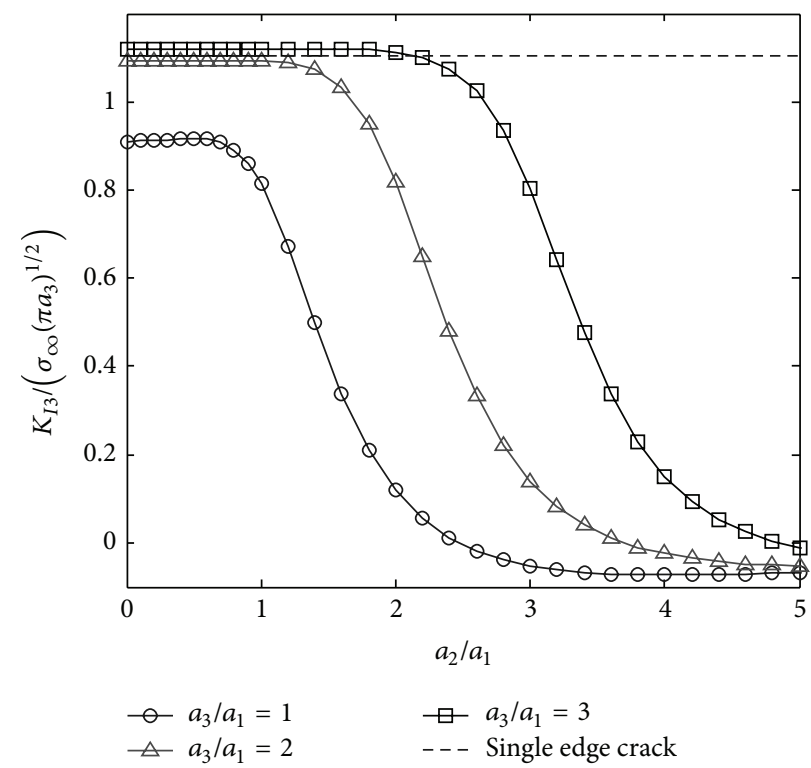

(c)

FIGURE 6: Nondimensionalized stress intensity factor of three cracks in a semi-infinite domain.

the $i$ th crack tip. When $g(\mathbf{X})<0$, the semi-infinite domain is considered to be failed. It should be noted that the value of $K_{l i}(\mathbf{X})$ is determined based not only on the information of the $i$ th crack but also on that of the other cracks such as the number, position, and length of those cracks. Therefore, $\mathbf{X}$ includes not only the information related to the $i$ th crack but also that of the other cracks.

To estimate the probability of the failure in a semi-infinite domain, the Monte Carlo simulation is adopted despite of its limitation in computational costs, because it can easily handle the change of the interaction of multiple cracks due to the randomness in $\mathbf{X}$ in (6). Other component-level probability evaluation methods such as the first-order and secondorder reliability methods [21] cannot effectively consider this change.

The repeated evaluation of a limit state in the Monte Carlo simulation considering the random lengths of cracks has been carried out on the basis of an automated finite element modeling procedure. The random crack lengths are generated using a random number generator in MATLAB, and then a finite element mesh is automatically generated as described in Section 2. After obtaining the stress intensity factor from the finite element analysis with ABAQUS, the limit state function in (6) has been evaluated by plugging in those 


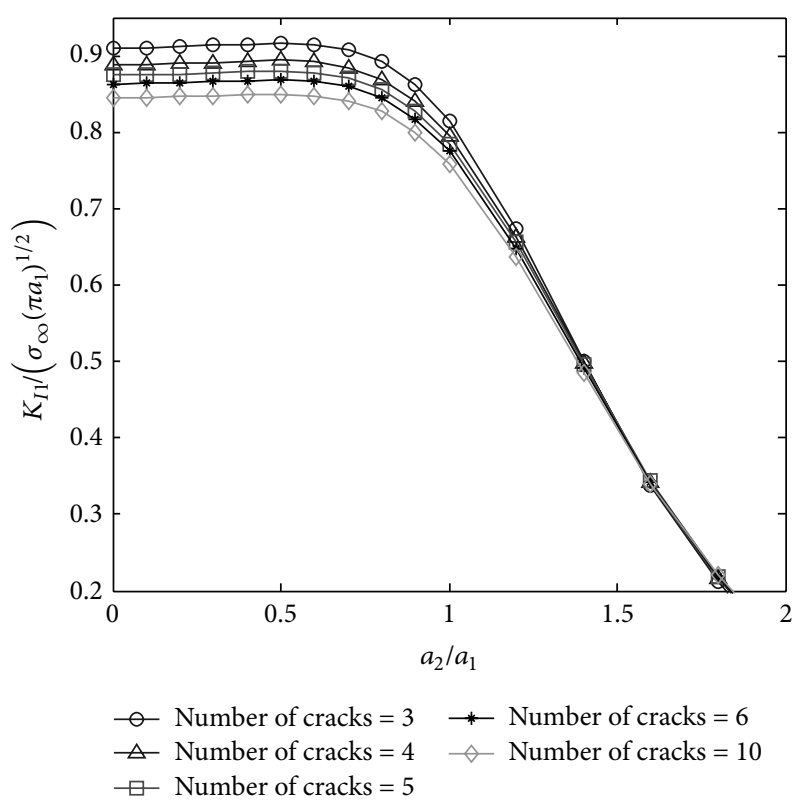

FIGURE 7: Effects of number of cracks on the stress intensity factor.

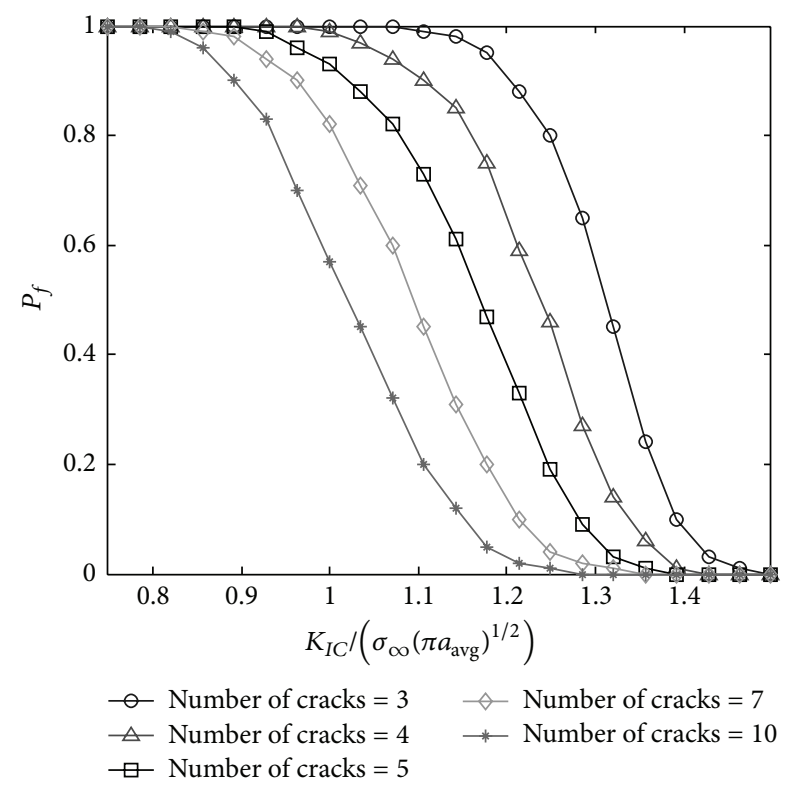

FIGURE 8: Failure probability of a semi-infinite domain with multiple cracks when the crack lengths vary gradually.

lengths to check if the domain fails according to the condition that $g(\mathbf{X})<0$. Note that the random length is defined as a truncated normal distribution with the assigned mean and standard deviation values. For the truncated normal distribution, the minimum and maximum length ratios to the averaged crack length $\left(a_{\mathrm{avg}}\right)$ are taken as 0.1 and 2.0, respectively, to avoid any negative values or extremely small or large values. In each probability evaluation, 1000 random samples are generated that provide the coefficient of variation of about $30 \%$ for a probability of the order of $10^{-2}$. The required computation time is 6 hours with the combined use
TABLE 1: Failure probability of a semi-infinite domain with 3, 4, and 5 cracks.

\begin{tabular}{lccc}
\hline$K_{I C} /\left(\sigma_{\infty} \sqrt{\pi a_{\text {avg }}}\right)$ & 3 cracks & 4 cracks & 5 cracks \\
\hline 0.803 & 0.890 & 0.830 & 0.720 \\
0.892 & 0.510 & 0.350 & 0.270 \\
0.981 & 0.210 & 0.090 & 0.040 \\
1.070 & 0.050 & 0.010 & 0.000 \\
\hline
\end{tabular}

of ABAQUS and MATLAB on a computer with an Intel Core i3-2100@ $3.1 \mathrm{GHz}$ and 8 GB of RAM.

5.2. Results. When 3, 4, and 5 cracks exist, the failure probabilities of a semi-infinite domain are evaluated by varying the threshold values of a stress intensity factor, that is, fracture toughness $\left(K_{I C}\right)$. The normalized threshold values, that is, $K_{I C} /\left(\sigma_{\infty} \sqrt{\pi a_{\text {avg }}}\right)$, are chosen as $0.803,0.892,0.981$, and 1.070 to represent the changes in the material property. The spacing ratio to the averaged crack length $\left(s_{i j} / a_{\text {avg }}\right)$ is fixed as unity, and the ratio of the standard deviation to the averaged crack lengths $\left(\sigma / a_{\text {avg }}\right)$ is taken as 0.1 . The evaluated failure probabilities are shown in Table 1 . As expected, the failure probability increases as the threshold value increases demonstrating that a greater stress intensity factor is required to reach a failure state. As the number of cracks increases, the failure probability decreases when the threshold value is fixed. This is because the total stress generated by the remote tension to the semi-infinite domain is redistributed to all the cracks, as explained by the shielding effect among cracks.

The failure probabilities of a semi-infinite domain are evaluated when there are $3,4,5,7$, and 10 cracks. In this case, the mean values of each length ratio to the averaged crack length gradually increase from 0.5 to 1.5 . In other words, the far-left crack length has the ratio of 0.5 and the farright crack length has the ratio of 1.5, and the lengths of the intermediate cracks are linearly interpolated based on the lengths of the two far-end cracks. The distance ratio between two adjacent cracks is 1.0, and the standard deviation ratio of all the crack lengths to the unit crack length is fixed to be 0.1 . The results are repeatedly evaluated for the normalized threshold stress intensity factor varying from 0.749 to 1.463 . The evaluated failure probabilities $\left(P_{f}\right)$ are reported in Figure 8. It is seen from the figure that the failure probability decreases according to the number of cracks and the threshold stress intensity factors. This is consistent with the results in Table 1 where the mean values of all crack length ratios were fixed as 1.0 while the mean value ratios vary from 0.5 to 1.5 in the analyses in Figure 8. The shape of the plots is similar to the inverse of a normal cumulative distribution function that represents the distribution of a system event consisting of normal random variables with a deterministic threshold value. If we compare Table 1 and Figure 8, when the normalized critical stress intensity factor $\left(K_{I C} /\left(\sigma_{\infty} \sqrt{\pi a_{\mathrm{avg}}}\right)\right)$ is 1.070 , the probabilities in Table 1 are less than 0.05 but Figure 8 shows the values between 0.32 and 1.00 according to the number of cracks. This difference is because the longest crack ratio in the latter case has a mean value of 1.5 , which 


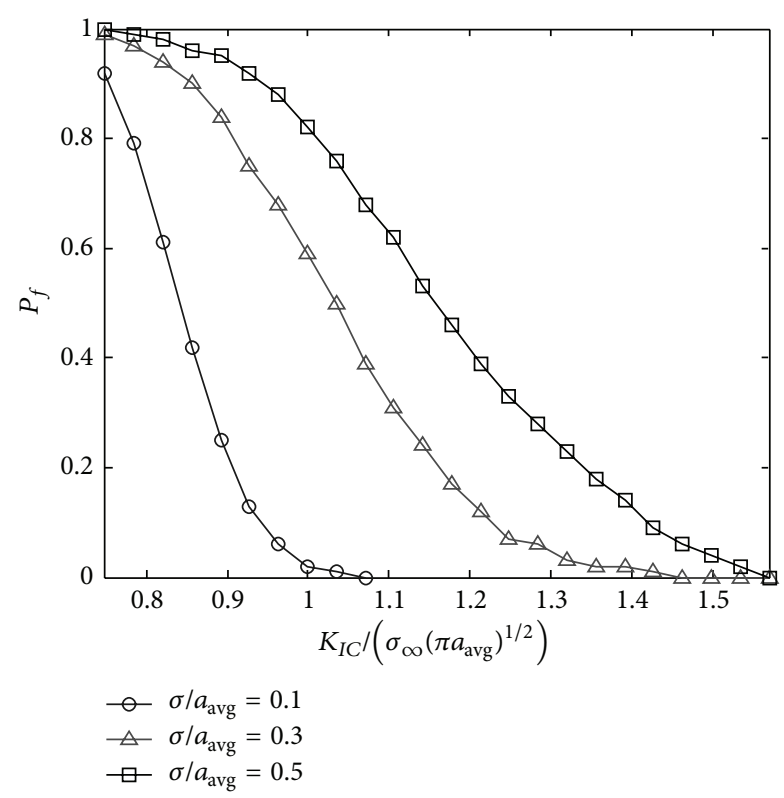

FIGURE 9: Failure probability of a semi-infinite domain with five cracks when the standard deviations of the cracks change.

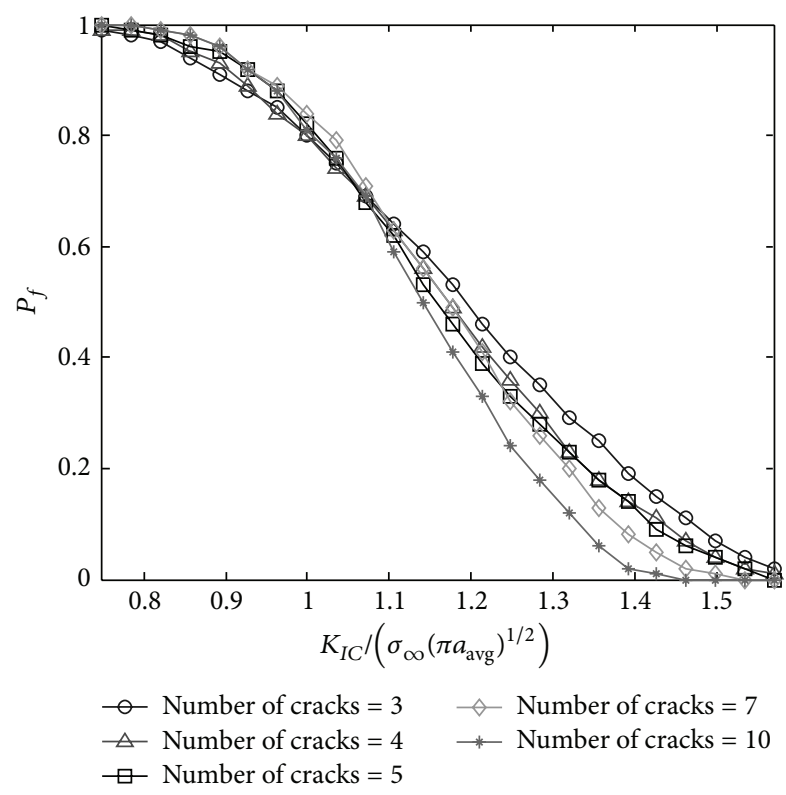

FIgURE 10: Failure probability of a semi-infinite domain with multiple cracks when the standard deviation of each crack length is fixed to be $5 \mathrm{~mm}$.

results in a significantly higher failure probability than a crack with a mean length ratio of 1.0 and causes a system failure with a high likelihood according to the system definition that the system fails if there is a failure at any crack tip.

The failure probabilities of a semi-infinite domain with multiple cracks are evaluated when the standard deviation of the crack lengths changes. The number of cracks is fixed to be 5 , and the mean length ratios and distance ratios between cracks to the averaged crack length are also fixed to be 1.0 for both. The three cases are considered and compared: the standard deviation ratio of all the lengths to the averaged crack length $\left(\sigma / a_{\text {avg }}\right)$ is equally (i) 0.1 , (ii) 0.3 , and (iii) 0.5 . The evaluated failure probabilities are plotted in Figure 9. It is seen from the figure that the failure probability increases as the standard deviation increases. This is because a greater standard deviation means a greater randomness in the lengths of the cracks, which reduces the crack shielding effect for the longest crack, and thus gives a higher chance of a violation of the limit state.

Figure 10 shows the failure probability of a semi-infinite domain with multiple cracks when the standard deviation ratio is fixed to be 0.5 . The mean length ratios and spacing ratios to the averaged crack length are fixed to be 1.0 for both. By comparing the results in Table 1 and those in Figure 10, a mixed effect of crack shielding effects and the increase uncertainties in crack lengths are observed as follows. In Table 1, the failure probability always decreases when the number of cracks increases, due to the crack shielding effect. However, in Figure 10, this trend is observed only when the normalized threshold stress intensity factor is greater than 1.1, and otherwise it is opposite. This is because the standard deviation ratio of the crack lengths has been increased from 0.1 in Table 1 to 0.5 in Figure 10, and more chances are given to the failure at each crack tip. If the number of cracks increases, the chance of the failure of at least one crack also increases. Therefore, this increase in the standard deviation ratio makes the opposite effect to the crack shielding effect, and according to the balance between the effect of uncertainties in crack lengths and the crack shielding effect, the failure probability is determined. The balance point is observed around the point where the failure probability is approximately 0.7 .

\section{Conclusion}

An investigation on the interaction effects of multiple parallel cracks in a semi-infinite domain was carried out deterministically and probabilistically. Multiple cracks in the domain resulted in stress redistribution, and thus the stress intensity factor changed according to the position, the length, and the number of cracks. Parallel cracks in an infinite domain and semi-infinite domain resulted in the decrease of the stress intensity factor because of the crack shielding effect. When the relative crack spacing was large enough, the effects of crack interactions decreased, which corresponded to a single crack example. The investigation on the multiple cracks in a semi-infinite domain was extended to a probabilistic study by considering the randomness in crack lengths. The series-system failure probability of a semi-infinite domain with multiple cracks was evaluated using the Monte Carlo simulation, and the randomness in the crack lengths was modeled using a truncated normal distribution. The effects of the number of cracks and the lengths of the cracks in terms of their partial statistical descriptors of the cracks lengths to the failure probability of a semi-infinite domain were investigated. Some observations were made as follows. (i) As the number of cracks increased, the failure probability was 
decreased due to the stress redistribution to all the cracks, which is called the crack shielding effect. (ii) The failure probability of a semi-infinite domain was mostly affected by the longest crack length instead of the average crack length of multiple cracks. (iii) The failure probability was determined based on the balanced effect of the uncertainty of the crack lengths and the crack shielding effect.

\section{Disclaimer}

The information presented in this paper is the sole opinion of the authors and does not necessarily reflect the views of the sponsoring agencies.

\section{Conflict of Interests}

The authors declare that there is no conflict of interests regarding the publication of this paper.

\section{Acknowledgment}

Dr. Park acknowledges support from Korea Agency for Infrastructure Technology Advancement (KAIA) through Grant no. 14CTAP-C078774-01.

\section{References}

[1] Y. Z. Chen, "Multiple crack problems for finite plate with arbitrary contour configuration," Engineering Fracture Mechanics, vol. 31, no. 2, pp. 289-295, 1988.

[2] Z. D. Jiang, A. Zeghloul, G. Bezine, and J. Petit, "Stress intensity factors of parallel cracks in a finite width sheet," Engineering Fracture Mechanics, vol. 35, no. 6, pp. 1073-1079, 1990.

[3] M. Isida, T. Yoshida, and H. Noguchi, "Parallel array of semielliptical surface cracks in semi-infinite solid under tension," Engineering Fracture Mechanics, vol. 39, no. 5, pp. 845-850, 1991.

[4] H. M. Shu, J. Petit, Z. D. Jiang, and G. Bezine, "Stress intensity factors and interaction of three cracks on both edges of finite width sheet," Engineering Fracture Mechanics, vol. 45, no. 3, pp. 407-414, 1993.

[5] D. K. L. Tsang, S. O. Oyadiji, and A. Y. T. Leung, "Multiple penny-shaped cracks interaction in a finite body and their effect on stress intensity factor," Engineering Fracture Mechanics, vol. 70, no. 15, pp. 2199-2214, 2003.

[6] S. Loehnert and T. Belytschko, "Crack shielding and amplification due to multiple microcracks interacting with a macrocrack," International Journal of Fracture, vol. 145, no. 1, pp. 1-8, 2007.

[7] M. Kamaya, "Growth evaluation of multiple interacting surface cracks. Part II: growth evaluation of parallel cracks," Engineering Fracture Mechanics, vol. 75, no. 6, pp. 1350-1366, 2008.

[8] T. Liu, P. Cao, and H. Lin, "Evolution procedure of multiple rock cracks under seepage pressure," Mathematical Problems in Engineering, vol. 2013, Article ID 738013, 11 pages, 2013.

[9] X. Xiaofeng, F. Yunwen, Y. Zhongwei, and F. Yuansheng, "Research on the plane multiple cracks stress intensity factors based on stochastic finite element method," Chinese Journal of Aeronautics, vol. 22, no. 3, pp. 257-261, 2009.

[10] E. D. Leonel, A. Chateauneuf, and W. S. Venturini, "Probabilistic crack growth analyses using a boundary element model: applications in linear elastic fracture and fatigue problems," Engineering Analysis with Boundary Elements, vol. 36, no. 6, pp. 944-959, 2012.

[11] G. Q. Feng, Y. Garbatov, and C. G. Soares, "Probabilistic model of the growth of correlated cracks in a stiffened panel," Engineering Fracture Mechanics, vol. 84, pp. 83-95, 2012.

[12] M. S. Chowdhury, C. Song, and W. Gao, "Probabilistic fracture mechanics with uncertainty in crack size and orientation using the scaled boundary finite element method," Computers \& Structures, vol. 137, pp. 93-103, 2014.

[13] G. E. Blandford, A. R. Ingraffea, and J. A. Liggett, "Twodimensional stress intensity factor computations using the boundary element method," International Journal for Numerical Methods in Engineering, vol. 17, no. 3, pp. 387-404, 1981.

[14] R. S. Barsoum, "Triangular quarter-point elements as elastic and perfectly-plastic crack tip elements," International Journal for Numerical Methods in Engineering, vol. 11, no. 1, pp. 85-98, 1977.

[15] A. R. Ingraffea and C. Manu, "Stress-intensity factor computation in three dimensions with quarter-point elements," International Journal for Numerical Methods in Engineering, vol. 15, no. 10, pp. 1427-1445, 1980.

[16] C. F. Shih, B. Moran, and T. Nakamura, "Energy release rate along a three-dimensional crack front in a thermally stressed body," International Journal of Fracture, vol. 30, no. 2, pp. 79102, 1986.

[17] T. Belytschko and T. Black, "Elastic crack growth in finite elements with minimal remeshing," International Journal for Numerical Methods in Engineering, vol. 45, no. 5, pp. 601-620, 1999.

[18] J. T. Oden and C. A. Duarte, "Clouds, cracks and FEM's," in Recent Developments in Computational and Applied Mechanics, B. D. Reddy, Ed., pp. 302-321, International Center for Numerical Methods in Engineering, CIMNE, Barcelona, Spain, 1997.

[19] K. Park, J. P. Pereira, C. A. Duarte, and G. H. Paulino, "Integration of singular enrichment functions in the generalized/extended finite element method for three-dimensional problems," International Journal for Numerical Methods in Engineering, vol. 78, no. 10, pp. 1220-1257, 2009.

[20] J. R. Rice, "A path independent integral and the approximate analysis of strain concentration by notches and cracks," Journal of Applied Mechanics, vol. 35, no. 2, pp. 379-386, 1968.

[21] A. H.-S. Ang and W. H. Tang, Probability Concepts in Engineering Planning and Design, Wiley, 2007. 


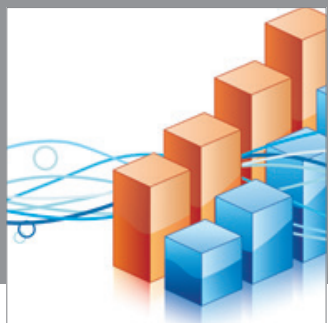

Advances in

Operations Research

mansans

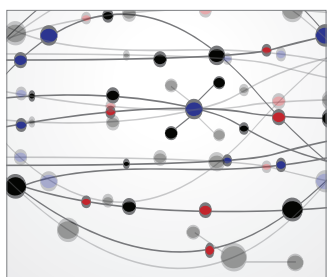

The Scientific World Journal
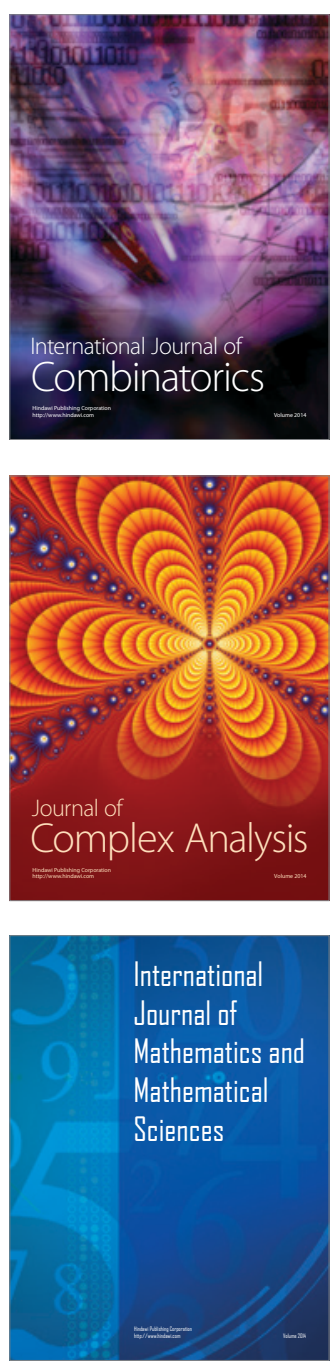
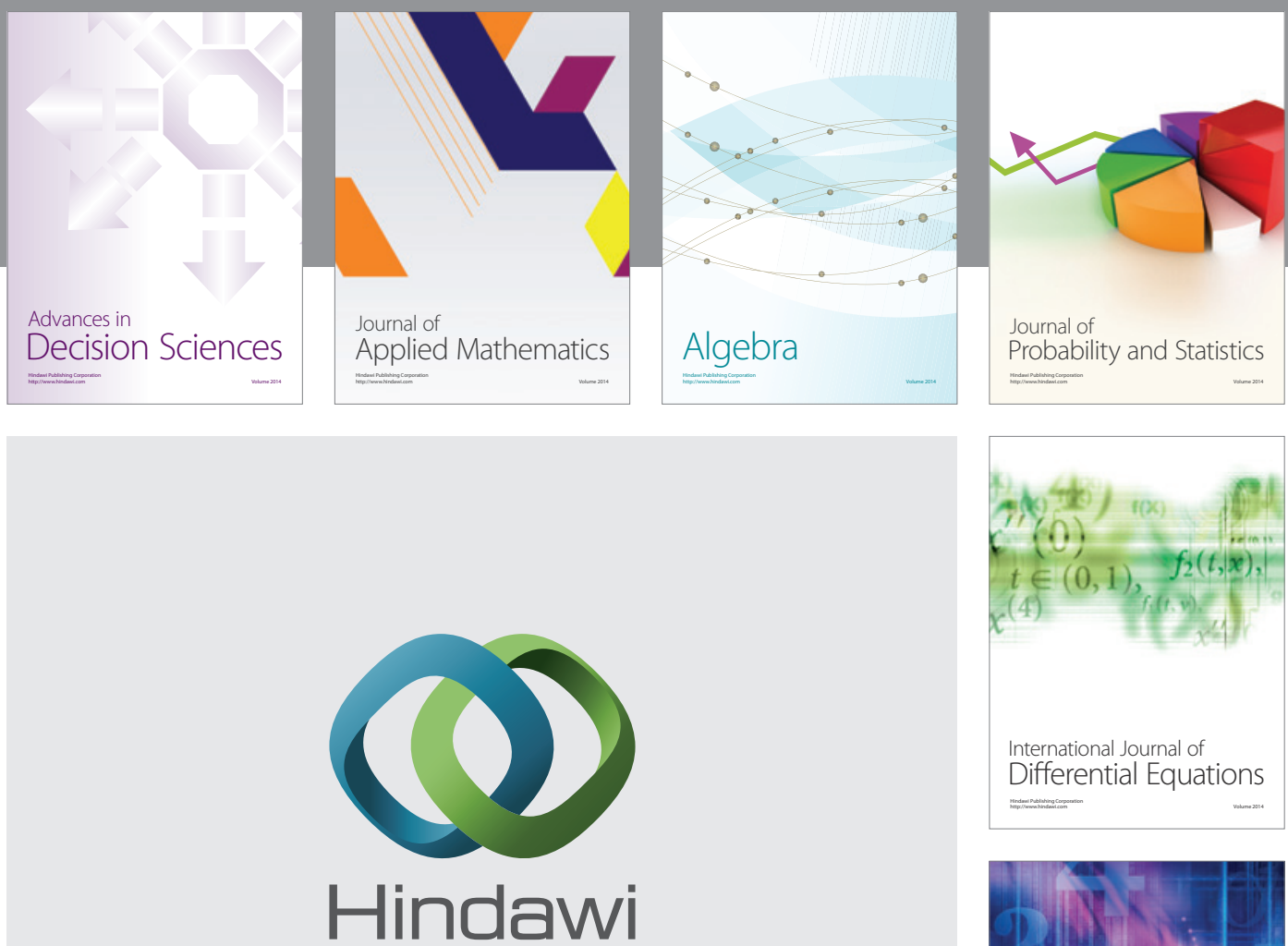

Submit your manuscripts at http://www.hindawi.com
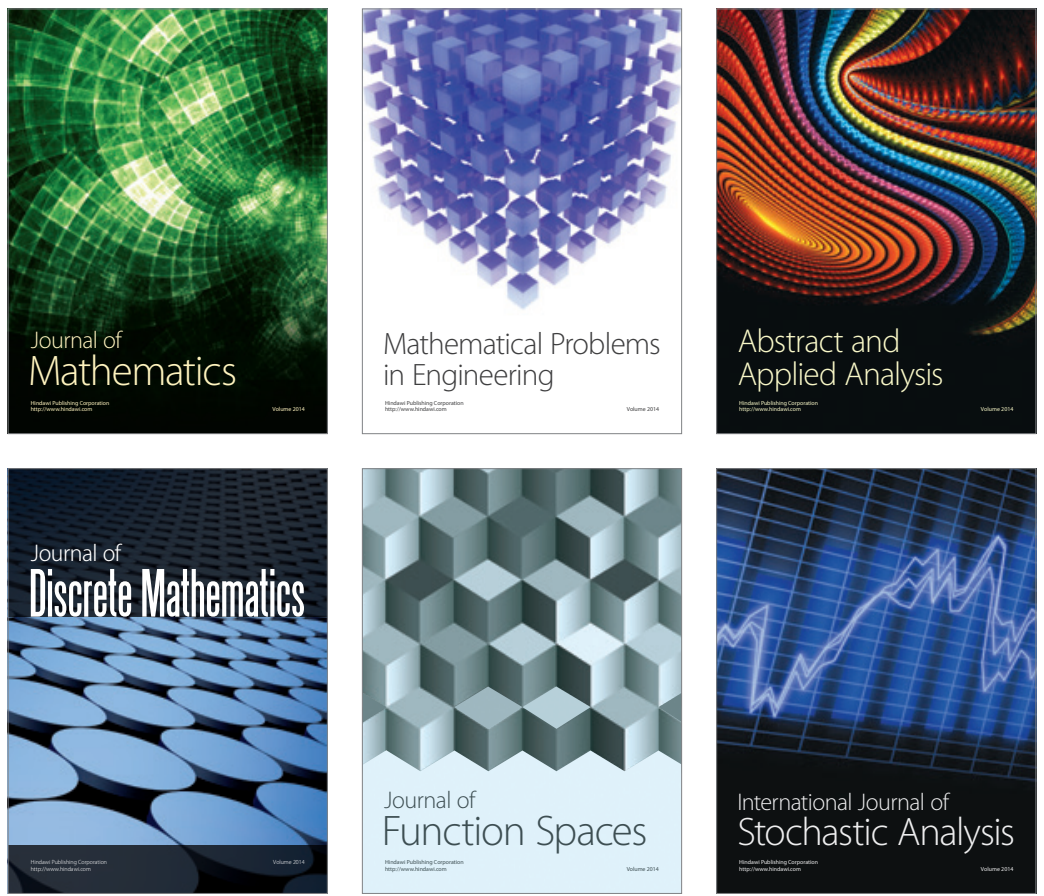

Journal of

Function Spaces

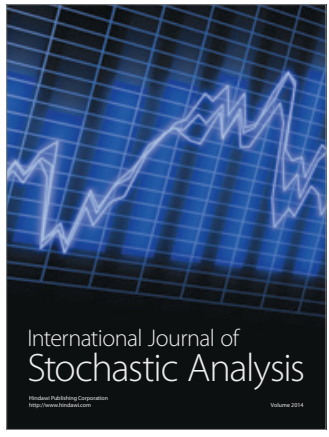

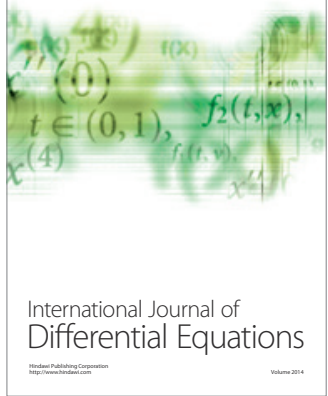
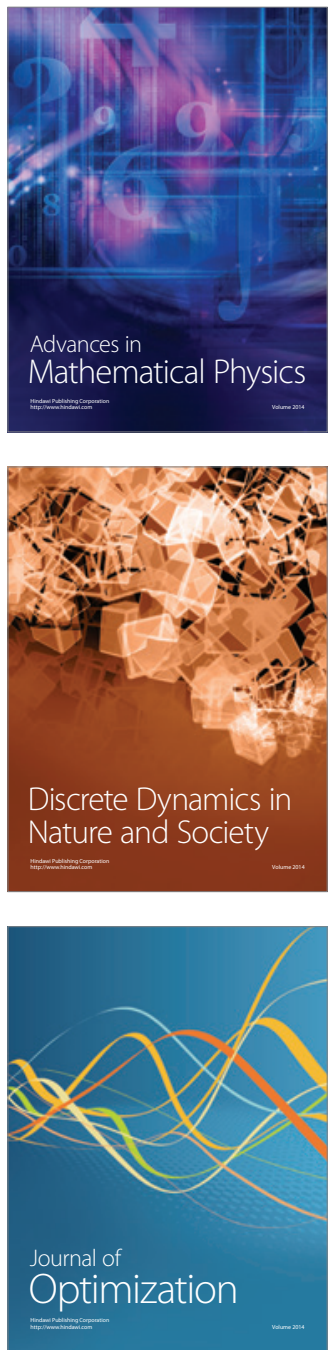